\title{
Mindfully implementing simulation tools for supporting pragmatic design inquiries
}

\author{
TIMO HARTMANN*, LEON OLDE SCHOLTENHUIS, VEDRAN ZERJAV ${ }^{\dagger}$ and \\ CARISSA CHAMPLIN
}

Construction Management and Engineering, University of Twente, Enschede 7500AE, The Netherlands

(Received 6 March 2014; accepted 26 October 2014)

\begin{abstract}
Based upon a conceptualization of the engineering design process as pragmatic inquiry, this paper introduces a framework for supporting designers and design managers with a better understanding of the trade-offs required for a successful implementation of simulation tools. This framework contributes to the field of technology implementation research by extending the four principles of mindfulness-accounting for novelty, alertness to distinctiveness, sensitivity to different contexts, and awareness of multiple perspectives - to the realm of implementing simulation tools within design organizations. At the same time, the framework contributes to engineering project organization theory, by providing a structured manner of organizing an engineering design effort that is resourcefully supported by simulation tools and the capabilities these tools offer. The paper illustrates these contributions by applying the framework to retrospectively analyse the implementation of a traffic simulation tool within the setting of municipal city engineering.
\end{abstract}

Keywords: design inquiry, mindfulness, model abstraction, performance, simulation.

\section{Introduction}

Increasingly, simulation models become available that abstract both the design situation and the predictions of how well several chosen design alternatives address the design problems. In this way, simulation tools, while offering less accurate answers, allow for the flexibility to experiment in design situations during which prototyping or mathematical modelling is no longer possible. Additionally, as engineering design efforts become more and more multi-disciplinary and globally distributed, simulation tools also provide the means to generate the required boundary and epistemic objects that only make collaborative design work possible in the first place (Henderson, 1991, 1999; Carlile, 2002; Ewenstein and Whyte, 2009). It is not surprising that the importance of supporting engineering design efforts with simulation is well acknowledged both in theory and in practice.

Despite this widely acknowledged potential, designers still often struggle with integrating the use of simulation tools into specific design processes. For example, often, much time and effort are invested in simulation modelling and output evaluation too early in the design process when detailed simulation outputs are not yet required. At the same time, in later design stages, where detailed simulation experiments could provide meaningful decision support, simulation is often not used because of the stringent time and resource constraints in these phases. As with all technological innovations, the implementation of simulation tools within the organizational processes needs to be mindful (Swanson and Ramiller, 2004) in the sense of being grounded in the specifics and facts of the design process at hand so that the tools can support and streamline design-related decisions.

To help designers become more sensitive to the specific requirements for the well-balanced integration of simulation tools in design processes, or, in other words, to help designers become more mindful, this paper introduces a framework that integrates the different technological trade-offs that must be made during

\footnotetext{
*Author for correspondence. E-mail: t.hartmann@utwente.nl

${ }^{\dagger}$ Current address: Bartlett School of Construction and Project Management, University College London, 1-19 Torrington Place, London WC1E $7 \mathrm{HB}, \mathrm{UK}$

(C) 2014 Taylor \& Francis
} 
the use of simulation tools with the process-related trade-offs that must be made during design processes. Based on the depiction of the engineering design process as pragmatic inquiry (Dewey, 1938), the framework intends to create awareness about the necessary trade-offs required for the implementation of a specific simulation software. Specifically, the framework allows designers to account for the inherent novelty characterizing every design process, the resulting distinctiveness of the process, and for different perspectives of the actors involved within the process. This awareness, in turn, will then allow for implementing simulation tools more mindfully in design practice as it allows the designer to understand the specifics of his/her design project context in relation to the features of the simulation software. This understanding may facilitate a better introduction of the simulation tool into their design-related decision-making processes.

We proceed as follows: The paper starts by summarizing work in mindful technology implementation, arguing that this work is also relevant to understand the implementation of simulation tools within design processes. We then introduce the design process by describing it as pragmatic inquiry (Dewey, 1938). Based on this depiction of the design process, the paper continues by introducing the framework to support mindful decisions about the introduction of simulation tools within design processes. We then illustrate the framework by retrospectively analysing the implementation of a simulation tools in a design effort that we followed in a previous research effort. We finally conclude with a discussion of the practical and theoretical implications of the presented work.

\section{Organizational mindfulness during the implementation of technologies}

Originally, the concept of mindfulness was developed as a psychological characteristic of individuals who are open to novelty, alert to distinction, sensitive to different contexts, implicitly, if not explicitly, aware of multiple perspectives, and oriented in the present (Sternberg, 2000). In a number of seminal papers, Karl Weick, together with a number of colleagues, extended the concept of mindfulness from individuals to organizations (Weick and Sutcliffe, 2001; Weick et al., 2008). Within this work, mindfulness is depicted as a characteristic of organizations that is mainly, but not completely, grounded in the ability of the organizations' members to interrelate meaningfully (Weick and Roberts, 1993). Through mindful interrelation, individuals can interpret and act upon a model of their organizational situation that allows them to coordinate their individual actions in accordance with their complementary representation of the situation in its organizational environment.

Originally, the concept of organizational mindfulness was widely used to analyse highly reliable organizations that operate in 'unforgiving social and political environments' (Weick et al., 2008, p. 83), for example, to explain dynamics around nuclear power plants, aircraft carriers and woodland fire-fighters. More recently, the concept of mindfulness has been extended to other kinds of organizations that are less prone to crisis situations (Weick et al., 2008). Examples for such organizations are hospitals (Vogus and Sutcliffe, 2007) and software firms (Vogus and Welbourne, 2003). Another area where the principle of mindfulness has been applied is the construction industry to understand safety (Mitropoulos and Cupido, 2009) or construction and operations performance (Brady and Davies, 2010). As a concerted venture into the unexpected, every engineering design process is, in this view, a potentially critical area for mindful behaviour. This is particularly the case when the design process is to be supported with advanced simulation technologies.

The application of simulation within the design process can be seen in itself as a mindful reaction to deal with emerging uncertainties through the targeted evaluation of different possible scenarios. At the same time, efforts to experiment with the use of simulation tools may themselves be more or less mindful. This dual role requires engineering design organization to foster the effective application of the simulation tool. During such application processes, mindful behaviour will not simply entail the promotion of all technical possibilities to evaluate scenarios in all their intrinsic detail. Mindfulness equally needs to promote wariness (Weick et al., 2008) that provides a resistance to jumping on all the technological possibilities modern simulation methods offer.

To support designers with such mindful implementations, in this paper, we attempt to derive a framework that can foster such an appreciation. Because the final goal of implementing simulation tools is the support of design processes, we begin this undertaking with an examination of the general nature of the design process itself.

\section{Pragmatic design inquiry}

This paper follows the long-standing tradition to depict the design process as pragmatic inquiry also often termed as problem-based inquiry. There is a large degree of convergence between the design thinking literature and pragmatic inquiry as it was developed by Dewey (Dewey, 1938). As such, pragmatism offers a 
'well developed and coherent articulation of concerns that are central to design thinking' (Dalsgaard, 2014). Therefore, the theory of pragmatic inquiry lends itself well to develop the framework for supporting designers to mindfully integrate simulation tools into design processes that this paper introduces. This section continues with a brief introduction of pragmatic inquiry to set the stage for the subsequent development of the framework.

All pragmatic inquiry begins with doubtful situations (Dewey, 1938, p. 105). For example, during civil engineering design, it is usually the situation of the existing built environment and doubts about how well the existing built environment can support the economic and social life of its inhabitants that triggers design inquiries. The aim of civil engineering design inquiries is then to develop plans for how to transform the existing built environment into one that resolves the doubtful situation. To this end, in a first aspect of the inquiry process, designers identify and explore problems (Dewey, 1938, p. 107). Next to this aspect of problem formulation, designers then develop ideas of how to transform the existing situation to address the previously identified problems. In other words, designers develop alternatives for a meaningful transformation of the existing environment to address the previously identified problems. A final aspect of pragmatic design inquires is then experimentation-designers have to conduct experiments to learn how well different alternatives address the problems. This experimentation aspect allows them to compare the different alternatives and to choose a final design alternative to transform the existing built environment.

In practice, each of these basic aspects of the design inquiry process highly influences each other. Knowledge that designers acquired during one of the aspects serves as important input for the other aspects. During problem formulation, designers get ideas for possible alternatives to transform the environment. At the same time, they develop experimental knowledge about how alternatives address the problem under development. During the generation of alternatives, designers gain new knowledge about the meaning of certain problems of the doubtful situation. At the same time, it is impossible to generate alternatives without already developing experimental knowledge of how the alternatives address the problem. Finally, during experimentation, designers develop new knowledge about the meaning of the problems and, at the same time, ideas will arise for alternative ways to address these problems. Therefore, the above-described process of design inquiry is highly iterative with an interactive interplay between the phases of problem formulation, alternative generation and experimentation.

Without a well-formulated problem, designers might develop solutions that address inadequate problems in the sense that their solutions do not have the potential to overcome the doubtful situation in the environment. The more ideas designers develop to transform the existing environment, the more likely it will be that they find a solution that is most optimal for a successful transformation of the doubtful situation. Finally, the better they model suggested alternatives and the more thoroughly they experiment, the more likely they are able to choose the most adequate of the previously developed alternatives. However, the goal of the inquiry process is to develop a feasible plan for a physical transformation of the existing environment. It is, therefore, important that designers manage design inquiries as effectively as possible by thoughtfully balancing the effort they spend on each of the three inquiry aspects. Figure 1 shows a sketch that illustrates these necessary trade-offs.

Ultimately, designers must make decisions about how to perform each of the above aspects based on decisions about the number of problems they want to address with any given detail. They also need to decide how many alternatives they want to experiment with, and how detailed these experiments should be. The next section describes how designers can support such decisions by accounting for the characteristics of simulation tools in relation to the specifics of a design process at hand.

\section{Integrating simulation with pragmatic design inquiry}

In the previous section, we suggested that designers adjust how they use simulation with the trade-offs they need to make to balance the effort spent on problem formulation, alternative generation and experimentation. The effort they spend on experimentation should determine the time that designers allot for the input of information about alternatives and problems for the simulation run itself, the so called pre-processing, and the evaluation of the simulation outcomes, the so called post-processing (Augenbroe, 2002, p. 8).

The effort necessary for pre-processing, simulation, and post-processing, in turn, depends on the simulation's characteristics, in particular, on the amount of time needed for pre- and post-processing. Additionally, the time required for running a simulation forms another boundary condition. Based on these computational characteristics, designers need to make an informed choice between extremes in two dimensions. This is because simulation experiments abstract both the situation of the alternative and how the alternative addresses the design problems. On the one hand, designers have to decide about the level of detail of 


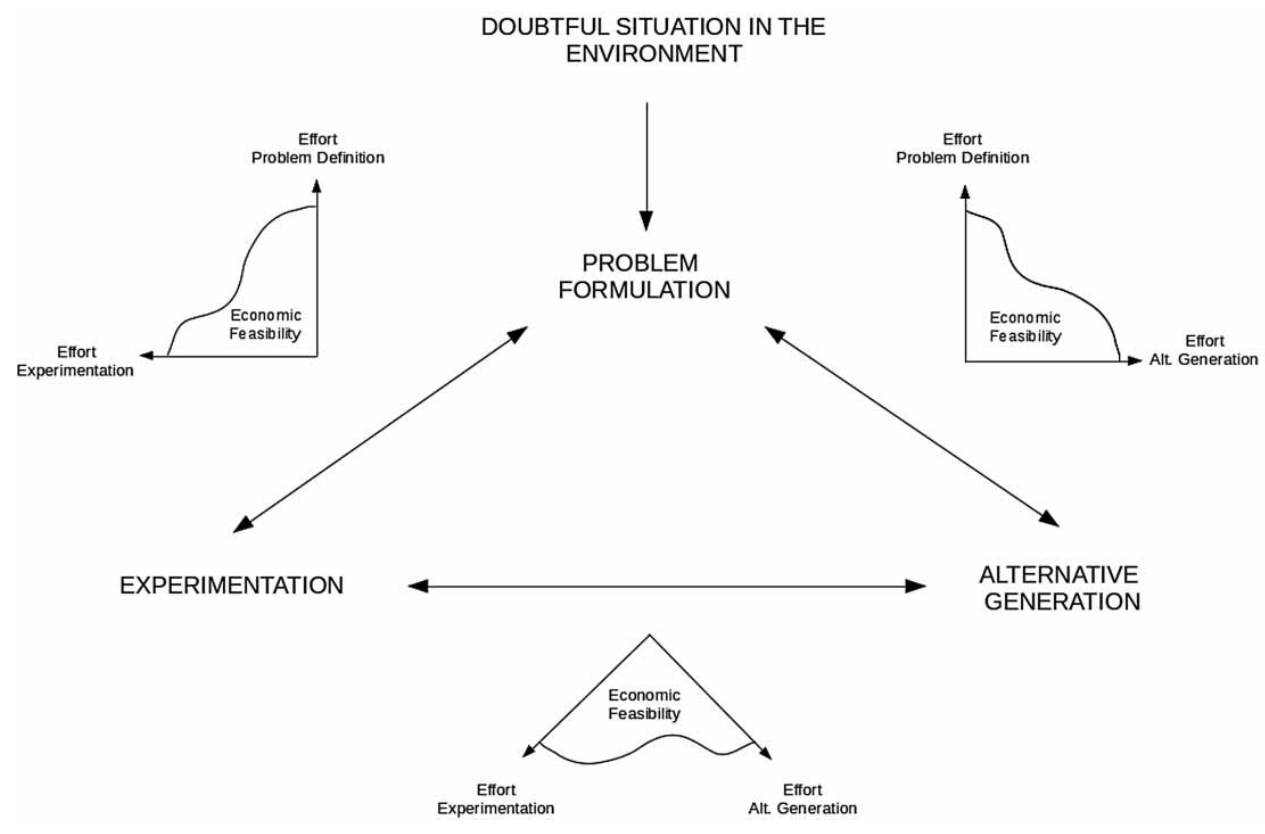

Figure 1 Trade-offs designers need to make to balance the aspects of problem formulation, alternative generation and experimentation during design inquiries

modelling the underlying structure of each alternative to be simulated (Küppers, 2006). On the other hand, designers must decide about the detail of the functional problem formulation that is to be assessed in the simulation (Küppers, 2006). Next to the decision of how to best balance structural and functional modelling efforts, designers also need to decide about the number of alternatives and problems they want to simulate. Figure 2 shows a sketch that illustrates the trade-offs designers need to make during the application of simulation tools.

Dependent on the computational feasibility of the available simulation tools and the time designers allocate for experimentation, the two above choices force designers to again make decisions about the number of alternatives and the details of these alternatives if they intend to implement a simulation tool mindfully. Designers can, for example, simulate a few, albeit very detailed, alternatives and evaluate how these alternatives address a large number of problems, or they can simulate a large number of alternatives and evaluate how they address one single very detailed problem. In any case, designers need to closely align the choices they make concerning how to balance problem formulation, alternative generation and experimentation during their design inquiry effort with the choices available to support their inquiry with simulation tools. This is done by balancing how much effort to spend
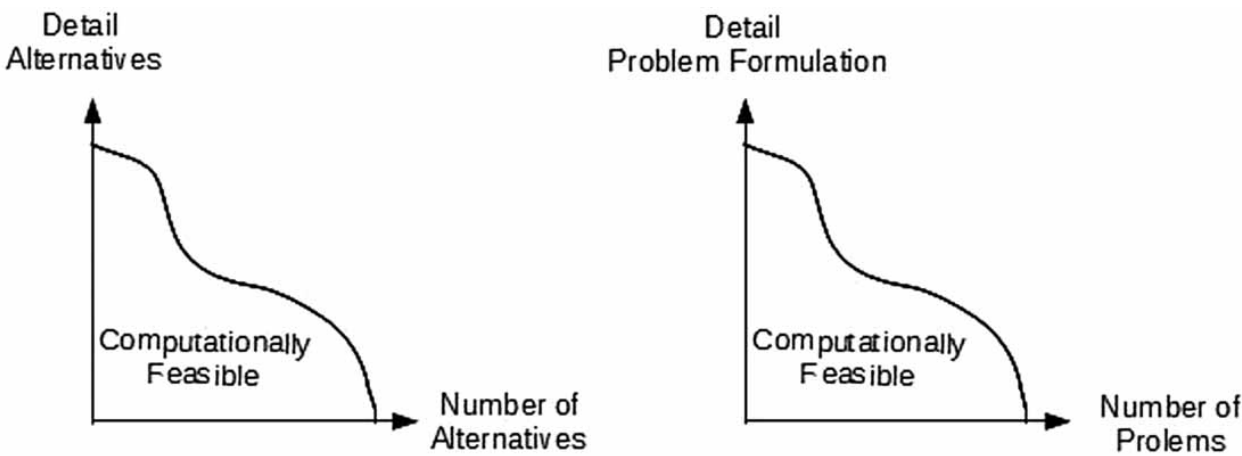

Figure 2 Trade-offs designers need to make during the application of simulation tools. The available computational and human resources will require a balance between how detailed problems are simulated and the number of problems simulated, as well as how detailed alternatives are modelled and how many alternatives are modelled 
on experimentation with what is computationally feasible.

In the end, a final well-balanced decision will significantly depend on the organizational design context in which the simulation tool is to be implemented. The above-sketched framework can help with making such decisions because it allows designers to explicitly understand the required trade-offs they must make. This, in turn, allows for the mindful implementation of the tool considering all four characteristics of a mindful process-accounting for novelty, alertness to distinctiveness, sensitivity to context and awareness of multiple perspectives (Swanson and Ramiller, 2004). The next section will illustrate this hypothetical potential by retrospectively analysing the degree of mindfulness during the implementation of a simulation tool on a recently conducted case study of a design organization.

\section{Illustration of the framework-designing an inner city traffic network to cope with construction activities}

\section{Case study method}

To illustrate the above framework, we retrospectively traced the successful integration of a simulation tool in the planning activities for inner city traffic of a midsized Dutch city. The introduction of the traffic simulation tool was conducted by a researcher-practitioner team that consisted of one researcher from a local university (the second author of this paper), one software developer from the provider of the simulation company, one Associate Professor from the local university (the first author of this paper) and one outside consultant who was involved in the initial development of the simulation tool. In the rest of this section, we will refer to this team as the simulation modelling team. To integrate the simulation tool in the planning processes, the simulation modelling team used ethnographic action-research methodology (Hartmann et al., 2009). The team worked in small iterative implementation steps that started by observing and understanding the needs of the local project. Based on the identification of these needs, the team then custom tailored the functionality of an existing traffic simulation software. The team finally integrated the adjusted software application in the project's design process and started another iterative action research cycle with new ethnographic observations of the projects design team.

This paper traces the implementation effort on the project and analyses the successful implementation using the papers framework as analytical lens. To this end, next to personal observations of the first author and second author that were jotted down in a notebook, we used data that the second author had collected and compiled in a number of reports. These reports summarized the researcher's observations on the project and analysed the implementation and application of the simulation tool. These research reports allowed us to understand the occurrences on the project from the angle of the presented framework. In this way, the report generation proved to be a valuable substitution for a more structured data collection and analysis effort that was not possible due to the evolving nature of the supporting activities on the project (Jorgensen, 1989). The next section describes the implementation of the simulation tool on the project in detail, starting with a description of the overall project background.

\section{Background}

The need for this change in the existing traffic structure was caused by the plans of this city to significantly revitalize its centre and to execute four big construction projects between 2010 and 2015:

- the renovation of the city's central hospital,

- the construction of a new hotel and apartment tower next to the hospital,

- the construction of a new entrance to the city's large-scale underground parking garage and

- the realization of an underground pedestrian tunnel that links the existing parking garage to the new hospital.

As the four projects were planned to occur almost simultaneously, city planners had to rethink the overall traffic routing within the centre to develop flexible design solutions to mitigate the adverse effects of the planned large-scale construction works on the traffic flow within the centre and the accessibility of it. This presented a novel design problem that the planners had not been confronted with and the design team had to be aware of. The design process started in early 2009 with the hospital initiating an early study to analyse the impact of the planned construction work on the inner city traffic. It was decided to support this effort with a traffic simulation tool.

\section{Implementation of the simulation tool}

After making the initial decision to support the design with simulation, the team had to choose an appropriate solution among the widely available traffic simulation tools. As the framework would have prescribed in this case, during this decision, the planning team first had to evaluate which of the different aspects of the pragmatic inquiry process to focus on. At this stage of the project, the problem was already relatively well 
formulated. In previous efforts, the city planners had conducted an in-depth stakeholder analysis effort. Fourteen different stakeholders, among others the program manager of the hospital construction project, different planners from the municipality and representatives of the local business owners, were interviewed about specific problems that were to be expected during the planned construction work. These problems were categorized and relations between them were mapped. This identification, understanding and categorizing led to five high-level objectives that the new traffic routing plan needed to fulfil: preserve accessibility to the city centre, reduce nuisance to citizens, do not disturb construction activities, minimize construction time and preserve spatial quality. All in all, the thorough analysis of the stakeholder context allowed the design team to account for the different contexts and perspectives of the stakeholders, one important part of a mindful implementation process.

It became apparent that, at this stage, a simulation tool could support the process of alternative generation and evaluation more than it would support the process of problem formulation. Intuitively following the framework sketched within this paper, the team started to understand the level of detail that was required for the description of the problems and the alternatives to be evaluated. Accounting for the previously gained understanding of the different perspectives of the well-sketched problem context, it was decided to focus on only a limited number of problems closely related to this context, but to model these problems in great detail. In particular, the team decided that the simulation should calculate indicators for the saturation of the street network, the average travel time on the network, the number of existing road obstructions, the average travel speed on the network, the possible occupancy of parking garages and the additional travel distance due to construction work.

At the same time, accounting for the distinctiveness of the situation and for the rather open nature of the possible solutions for these problems, it was decided not to model the different possible alternatives for mitigating the problem context in much detail. Rather, it was decided that the simulation tool should allow for the rapid creation of a large number of distinct alternatives. The team then planned to quickly develop such alternatives within creative meetings and evaluate their performance on the indicators. It was decided that the scenario modelling features of the required simulation tool should simply allow for the quick modelling of new roads, the connection of roads and the possibility to block roads in one or both driving directions. It was also decided to focus the alternative modelling possibilities on a very small area around the inner city centre to further reduce the detail of the alternatives to be modelled and, in turn, increase possibilities to quickly generate alternatives.
In the end, the simulation modelling team's evaluation that accounted for the novel and distinct character of the design project resulted in a number of requirements for the simulation tool that specifically catered to the existing context and the different perspectives of stakeholders involved within this process: The simulation tool should allow for the calculation of six very specific indicators and it should allow for the easy and quick generation of a large number of alternatives, albeit in a low level of detail. For this purpose, the outside consultant together with the university's research team developed a custom-tailored simulation application that specifically fulfilled the requirements established.

After some evaluation, the design team realized that none of the existing simulation software products on the market would support these requirements. Consequently, the team decided to work together with a software development company that could provide custom-tailored traffic simulation functionality on an ad-hoc basis for the project and that was willing to work closely together with the designers. In the rest of the case description, we will simply refer to this evolving and custom-tailored solution as the simulation tool, despite the continuously evolving character of the software.

\section{Application of the simulation tool}

Consecutively, the second author used the simulation tool to model and evaluate a large number of design scenarios. Based on evaluating these scenarios and making some basic assumptions about the city centre and its traffic conditions, the modelling team then established four alternatives by choosing different points in time during which one or more construction project activities were planned at the same time based on four scenarios for the planned construction activities:

- Scenario 1: Only the construction activities for the new hospital are ongoing.

- Scenario 2: Construction of the new hospital and the hotel are ongoing simultaneously.

- Scenario 3: Construction of the new hospital, construction of the hotel and construction of the new entrance to the parking garage are ongoing simultaneously.

- Scenario 4: Construction of the new hospital and the construction of pedestrian tunnel are ongoing.

These scenarios were based upon the following specific assumptions about the effect of the planned construction work on the inner city traffic planning activities that were developed during the alternative modelling and evaluation effort: 
- To allow for the construction of the hospital, it is necessary to close the existing parking lot in front of the hospital.

- During construction work there will be an increased demand for parking for the existing underground parking garage under the city centre to accommodate construction workers.

- Construction-related traffic will increase the number of cars in the city's network.

- A number of roads will need to be closed to allow for construction activities.

Table 1 summarizes how each of these assumptions was modelled using the traffic simulation tool.

Because of the mindful set-up of the simulation tool that only accounted for a limited detail of the underlying alternatives, the calculation of the detailed indicators for each scenario only lasted around five seconds. This allowed for the real-time evaluation of different distinct alternatives without the need to wait for the simulation program to finish its calculations and, in turn, for the quick iterations towards the above-described alternatives.

After developing the above-described alternatives, the team presented the simulation model in two multi-stakeholder meetings. During the first meeting, the team discussed possible alternatives to reroute traffic in the city centre with the construction project manager of the hospital. The participants of the second meeting were two construction project team members, a parking expert from the municipality, a first-aid expert, a trauma staff member, and a logistics and security expert. During the two meetings, the simulation modelling team displayed the model on a projection screen and supported the meeting by navigating the simulation software and by quickly adjusting underlying assumptions to generate new scenarios based upon the five initially modelled alternatives.

Overall, during the two meetings, the application of the simulation generated and streamlined discussions. For example, during the first meeting, the visualizations of the adjustment of the model in real time and the simulation of road saturation created a discussion about two critical road sections in the simulation area.

Table 1 The different numbers of alternatives modelled and their respective problem formulations

\begin{tabular}{lrrrr}
\hline & Alt. 1 & Alt. 2 & Alt. 3 & Alt. 4 \\
\hline Parking spaces hospital & 0 & 0 & 0 & 0 \\
Increased parking demand & 280 & 525 & 610 & 485 \\
Additional cars in centre & 100 & 290 & 375 & 275 \\
Roads blocked & 2 & 2 & 3 & 3 \\
\hline
\end{tabular}

This discussion was triggered by the request of one of the meeting participants to remove one of the assumed road blocks in the model and instead close another road. The modelling team introduced this change in the simulation's structural model for another simulation run conducted immediately within the meeting. The outcomes of this simulation run showed a decrease in indicators such as travel time, average speed and extra travel distance. However, the saturation of other roads on the network increased significantly. After seeing the simulation, the project manager of the hospital and the planner from the municipality started discussing the length and number of roads that they should block.

After the application in the two meetings, the city's traffic engineers also used the simulation tool for drafting a consultancy report. In particular, while writing this report, they used the simulation to develop recommendations about how to set up a network of road signs that reroute the traffic around residential area during construction to reduce undesired nuisance for local residents.

In the end, it can be very well stated that the abovedescribed development and implementation of the simulation tool enabled the planners on the project to design a traffic plan for the city centre. Hereby, the simulation tool provided great help in experimenting with different alternatives. Additionally, it can also be stated that the project team made explicit decisions about how to best use the simulation tools along the lines of the framework introduced in this paper, of course, without having the framework available at the time of the design effort. These explicit decisions, in turn, allowed the team to mindfully implement the simulation tool, which might have led to its successful application. The following section discusses these findings in more detail.

\section{Theoretical implications}

If nothing more, the case shows that a mindful implementation along the lines of what the earlier presented framework would have prescribed allowed for the application of a simulation tool that went beyond simply accounting for previously made design decisions and improved the ongoing development of design alternatives. During the simulation modelling process on the case project, the modelling team started with understanding the required design decision to be made and adjusted the simulation software to directly support these decision-making processes. In this early stage, the planners were mainly interested in understanding a large number of different planning alternatives assuming a very focused set of problems for a 
limited number of different stakeholders. To adjust the simulation to the trade-offs of the design process, the simulation modelling team accordingly focused on establishing a simulation tool that simulates a few problems in depth for many not very detailed alternatives. By allowing such meaningful decision support on the project, the above case is an example for the mindful implementation (Swanson and Ramiller, 2004) of a simulation application by a design organization. The planning team was able to develop and implement the simulation tool while adhering to the four principles of mindful organizational conduct as presented earlier in the theoretical section of this paper:

- Accounting for the novelty of the planning situation around the city centre: The simulation modelling team on the project was open to novelty in a way that they did not assume that it would not be easily possible to support the project without adjusting the underlying functional and structural modelling possibilities of existing simulation tools. One can well state that the simulation modelling effort was oriented in the present.

- Being alert to the distinctiveness of this situation: The team distinguished between the needs of the different planning stakeholders with respect to functional and structural modelling. To this end, the simulation modelling team did not try to implement a simulation tool with all the technically available features. On the contrary, the implemented simulation tool relied on a very simple traffic simulation algorithm and simplified underlying functional models that were targeted specifically to the needs of the design situation at hand.

- Being sensitive to different contexts within this situation: The simulation modelling team took great care in adjusting all models closely to the level of detail the planning team required during their ongoing decision-making. This focus on the actual state of the planning process and the close alignment of the simulation tool to this state then allowed for the modelling of more alternatives within the time available to the designers.

- Being implicitly, if not explicitly, aware of multiple perspectives and orientations: The simulation modelling team showed great sensitivity to multiple perspectives, making concerted efforts to understand the different stakeholders.

Overall, the case shows that the framework can describe simulation implementations that fulfil all four characteristics of mindful technology introduction efforts (Sternberg, 2000, p. 12). The above-described implementation can be characterized by these qualities and, this, in turn, might have been an important antecedent for the successful implementation of the simulation on the project.

The findings also illustrate some technical characteristics that simulation tools should possess to allow for their mindful implementation. Much has been written about the need of engineering designers for flexible epistemic objects that allow for the meaningful development of new design knowledge (Ewenstein and Whyte, 2009) and flexible boundary objects (Henderson, 1991, 1999; Carlile, 2002) that allow for the meaningful communication of design rationales between different stakeholders. While most of this previous research focuses on how practitioners can best generate visual tools that integrate well into the design process, little attention is given to the introduction of more and more computer-based simulation tools. Considering that simulation tools are today one of the main technologies to create visual epistemic objects, this study contributes to the above discussion by providing a framework that helps with understanding and implementing simulation tools that can be adjusted flexibly to the needs of designers.

The case also shows that such a close adjustment of simulation technologies to project needs requires a different way of developing software. The traditional linear software development process during which programmers develop non-malleable software which is then implemented as a ready-made product in organizations cannot work. Designers need to be able to adjust simulation software to allow for making the necessary tradeoffs required to truly support specific design inquiries. This also requires new software development processes that allow programmers of software to work closely with users. Other iterative development frameworks are required, such as ethnographic action research (Hartmann et al., 2009), the methodology that the simulation modelling team applied on the presented case project.

Of course, this study is not free of limitations. At the outset, this paper only provides an illustration of the framework using a retrospective case study. Therefore, it is not surprising that we were only able to provide a first and at times limited illustration of the power of the introduced framework. Additional empirical studies are needed that can more clearly establish the theoretical claims made in this paper empirically. Additionally, future action research studies need to be conducted that test the potential of the framework to guide ongoing implementation efforts. More studies are also needed to provide evidence that can show the generality of the framework in other design settings, in particular, in such settings that intend to design technically more complex engineering products.

Another limitation is the reliance of the framework on the pragmatic inquiry of Dewey that depicts a rather 
linear, albeit cyclical, model of the design process. Such systematic models have been criticized by the literature in design thinking, triggered by the writings of Schön (Schön, 1983). In this study, we chose for such a linear description as a pragmatic way to develop a systematic framework that can support organizational design efforts. Future practice-based studies conducted on the background of the design thinking literature should explore the relevance of this linear model during real-world design efforts.

Finally, it is also possible to implement a simulation tools for supporting a given design process mindlessly, that is, without adhering to the four principles of mindfulness summarized above: being open to the novelty of the process, being alert to distinction within the process, being sensitive to different contexts of the process and being aware of multiple perspectives. Under these circumstances, an organization would simply choose one of the many abundant simulation tools available and apply it without much reflection upon the requirements and needs of the design process at hand. Such a mindless implementation might be very well appropriate for design organizations, for example, in cases where outside market pressures dictate the use of sophisticated simulation tools, while the internal capacity of the design organization would not allow for the required reflection and wariness to truly integrate the tool at hand in the design-related decision-making process. Seldom, however, will such a mindless implementation of a simulation tool improve the design-related decisions and support designers arriving at a better design (Swanson and Ramiller, 2004). Mindfulness in the sense of a nuanced appreciation of the context of the design task and the design organization at hand is crucial to meaningfully implement simulation within the design process.

\section{Conclusion}

This paper argued that designers need to make tradeoffs between how much effort they want to spend on problem formulation, alternative generation and experiment. These trade-offs designers make for the inquiry process should determine how they use simulation programs to support their experimentation activities. The main contribution of the paper is then the development of a framework to support designers with making these trade-offs by aligning general characteristics of simulation with characteristics of pragmatic inquiry during the design process. This in turn should allow designers to mindfully implement simulation tools in their design processes, accounting for the novelty of these processes, their distinct character and the different perspectives and contexts of the specific design situation. The paper provides a first empirical illustration for this utility of the framework.

If nothing more, the framework is a first step towards better understanding the use of simulation during the design process. Using the framework, designers can make mindful decisions about what kind of simulation functionality they choose to support their work and how to integrate the use of the simulation tools in their design process. Additionally, the understanding will help simulation developers to adjust their simulation software to the specific needs of designers.

In conclusion, we believe that the framework can also be a starting point for future research efforts with the intent to better understand the relation between simulation technology and the design process. Despite the intensive theoretical efforts to understand the design process itself (Cross and Cross, 2000; Pahl et al., 2007) and the technical development of simulation programs (Ross, 1990; Kelton and Law, 2000), little research exists that attempts to develop such an understanding and to develop avenues of how to best combine design processes and simulation technology. We suggest that researchers use our framework as a starting point for their empirical work with designers aligning these two fields of research.

\section{References}

Augenbroe, G. (2002) Trends in building simulation. Building and Environment, 37(8), 891-902.

Brady, T. and Davies, A. (2010) From hero to hubris-reconsidering the project management of Heathrows terminal 5. International fournal of Project Management, 28(2), 151-7.

Carlile, P.R. (2002) A pragmatic view of knowledge and boundaries: boundary objects in new product development. Organization Science, 13(4), 442-55.

Cross, N. and Cross, N. (2000) Engineering Design Methods: Strategies for Product Design, Vol. 58, Wiley, Chichester.

Dalsgaard, P. (2014) Pragmatism and design thinking. International fournal of Design, 8(1), 143-55.

Dewey, J. (1938) The Theory of Inquiry. Holt, Rinehart \& Wiston, New York.

Ewenstein, B. and Whyte, J. (2009) Knowledge practices in design: the role of visual representations asepistemic objects'. Organization Studies, 30(1), 7-30.

Hartmann, T., Fischer, M. and Haymaker, J. (2009) Implementing information systems with project teams using ethnographic-action research. Advanced Engineering Informatics, 23(1), 57-67.

Henderson, K. (1991) Flexible sketches and inflexible data bases: visual communication, conscription devices, and boundary objects in design engineering. Science, Technology \& Human Values, 16(4), 448-73.

Henderson, K. (1999) On Line and On Paper: Visual Representations, Visual Culture, and Computer Graphics in Design Engineering. The MIT Press, Cambridge, MA. 
Jorgensen, D.L. (1989) Participant Observation: A Methodology for Human Studies. Vol. 15, Sage, Thousand Oaks, CA.

Kelton, W.D. and Law, A.M. (2000) Simulation Modeling and Analysis, McGraw Hill, Boston, MA.

Küppers, G. (2006) Computer simulation: practice, epistemology, and social dynamics, in Lenhard, J., Küppers, G. and Shinn, T. (eds.) Simulation: Pragmatic construction of relatity. Springer, Dordrecht, pp. 3-22.

Mitropoulos, P. and Cupido, G. (2009) Safety as an emergent property: investigation into the work practices of highreliability framing crews. Fournal of Construction Engineering and Management, 135(5), 407-15.

Pahl, G., Wallace, K. and Blessing, L. (2007) Engineering Design: A Systematic Approach, Vol. 157, Springer, London.

Ross, S.M. (1990) A Course in Simulation, Prentice Hall PTR, San Diego, CA.

Schön, D.A. (1983) The Reflective Practitioner: How Professionals Think in Action. Basic Books, New York.

Sternberg, R.J. (2000) Images of mindfulness. Fournal of Social Issues, 56(1), 11-26.
Swanson, E.B. and Ramiller, N.C. (2004) Innovating mindfully with information technology. MIS Quarterly, 28 (4), 553-83.

Vogus, T.J. and Sutcliffe, K.M. (2007) The safety organizing scale: development and validation of a behavioral measure of safety culture in hospital nursing units. Medical Care, 45(1), 46-54.

Vogus, T.J. and Welbourne, T.M. (2003) Structuring for high reliability: HR practices and mindful processes in reliability-seeking organizations. Fournal of Organizational Behavior, 24(7), 877-903.

Weick, K.E. and Roberts, K.H. (1993) Collective mind in organizations: heedful interrelating on flight decks. Administrative Science Quarterly, 38(3), 357-81.

Weick, K.E. and Sutcliffe, K.M. (2001) Managing the Unexpected, Jossey-Bass, San Francisco.

Weick, K.E., Sutcliffe, K.M. and Obstfeld, D. (2008) Organizing for high reliability: processes of collective mindfulness. Crisis Management, 3, 81-123. 